\title{
Traduire
}

Ine autre perspective sur I t raduction

Revue française de la traduction

245 | 2021

Regards sur l'interprétation

\section{Précis de traduction et d'interprétation en milieu judiciaire}

Dorina Irimia

\section{(2) OpenEdition \\ 1 Journals}

Édition électronique

URL : https://journals.openedition.org/traduire/2420

DOI : 10.4000/traduire.2420

ISSN : 2272-9992

Éditeur

Société française des traducteurs

Édition imprimée

Date de publication : 5 décembre 2021

Pagination : 27-39

ISSN : 0395-773X

Référence électronique

Dorina Irimia, « Précis de traduction et d'interprétation en milieu judiciaire », Traduire [En ligne], 245 |

2021, mis en ligne le 05 décembre 2021, consulté le 23 décembre 2021. URL : http://

journals.openedition.org/traduire/2420; DOI : https://doi.org/10.4000/traduire.2420 


\section{Précis de traduction et d'interprétation en milieu judiciaire}

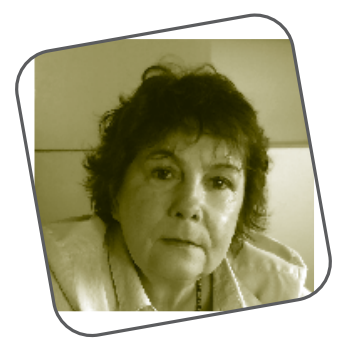

Dorina Irimia

La traduction et l'interprétation en milieu judiciaire sont deux pratiques différentes exercées dans un même domaine, celui de la justice. Nous examinons ici la situation des experts traducteurs-interprètes.

\section{Points de convergence entre traduction et interprétation en milieu judiciaire}

\section{L'urgence}

La justice française d'aujourd'hui est une justice dans l'urgence (Bastard et Mouhanna, 2007). Le traducteur doit en être bien conscient lorsqu'il reçoit une demande. Certains actes sont particulièrement urgents, notamment les mandats d'arrêt européen ou d'extradition, les ordonnances et les commissions rogatoires internationales de juges d'instruction qui sont à exécuter rapidement pour permettre l'avancement de la procédure, d'autant plus lorsque les personnes concernées se trouvent en détention provisoire. Mentionnons également les convocations à l'audience pour citer les prévenus. Un prévenu détenu est immédiatement remis en liberté 
si le tribunal correctionnel n'a pas commencé à examiner son affaire à l'expiration d'un délai de deux mois à compter de la date de l'ordonnance de renvoi du juge d'instruction au tribunal correctionnel.

Il arrive parfois qu'un procureur appelle lui-même un traducteur pour la traduction d'un mandat d'arrêt européen' (en exemple, il y avait à exécuter l'arrestation d'une personne lors de l'embarquement à l'aéroport à l'étranger et il restait deux heures au traducteur pour rendre sa traduction du mandat qui devait être transmis aux autorités étrangères). II arrive même qu'un officier de police judiciaire se déplace au domicile du traducteur pour la traduction très urgente d'un acte intéressant une commission rogatoire internationale.

Ces dernières années, tout particulièrement, et ce afin de répondre aux impératifs de la justice, les magistrats et les parquets ont imparti aux traducteurs des délais courts pour le dépôt de leurs traductions ${ }^{2}$. II s'ensuit que l'urgence est devenue un enjeu essentiel dans leur travail et que, pour pouvoir l'exécuter dans les délais impartis, ils doivent avoir suivi une solide formation.

Quant à l'interprète, il peut devoir se déplacer de jour comme de nuit pour répondre à diverses missions de la police, et ce, parfois loin de son domicile.

\section{L'adaptation constante à la mission}

Cette question a tout son intérêt, notamment lors des audiences correctionnelles de la cour d'appel et de la cour d'assises. L'interprète fait figure d'intervenant ad hoc, appelé si nécessaire lors d'une audience pénale. Toutes les audiences ont en commun que le juge doit rationaliser l'ordre du jour dans le prétoire et que le temps est limité sous la pression des audiences suivantes. Le rythme de l'intervention est très accéléré pour l'interprète; pour être efficace, il lui est indispensable d'avoir déjà quelque temps de pratique (on perçoit rapidement celui qui n'a jamais fait ou n'a fait que rarement

1. Un conseil: préparez à l'avance une version Word de ce texte traduit dans votre langue. Il existe au format PDF sur le portail de l'Union européenne.

2. Pour de plus amples informations, voir Irimia, 2021. 
des missions d'interprétariat). Comment procéder? II a le choix entre deux méthodes: la consécutive et la simultanée.

L'interprétation simultanée s'adapte au rythme soutenu, l'interprète suit le locuteur et traduit simultanément. Cette méthode convient davantage aux audiences pénales parce qu'elle est plus fluide et ne génère pas de perte de discours. Elle est également plus fidèle, dans la mesure où elle laisse moins de marge à l'interprète pour améliorer ou filtrer le message du prévenu (ce que les avocats n'apprécient guère). La méthode consécutive se prête moins à ce contexte, car elle demande plus de temps. Elle peut être incomplète (l'interprète perd des détails du discours), plus subjective, moins fidèle. Il n'est pas sûr que le juge l'accepte, la simultanée étant la règle.

Le choix entre la simultanée et la consécutive ne résout pas tout: il faut encore connaître la logique de la procédure et son déroulement. L'interprète confirmé s'en sort mieux que celui dont les missions sont rares. II semble ainsi judicieux, si l'on n'a pas la pratique, d'assister en auditeur libre à des audiences correctionnelles et d'y prendre des notes.

Il faut par ailleurs toujours veiller à rester dans la stricte limite de sa mission (ne rien ajouter ni ne rien omettre). Les avocats n'apprécient pas que l'interprète, s'il n'est pas satisfait des réponses de la personne, pose des questions supplémentaires. Néanmoins, les avocats ignorent souvent que l'interprétation implique aussi un sens pédagogique de la part de l'interprète (il aide la personne à formuler ses propos et, par conséquent, forcément, dialogue avec elle). Enfin, l'interprète doit se détacher de sa mission puisque, si celle-ci est trop complexe, il peut parfois se trouver malmené lui-même.

Si la simultanée est bien adaptée aux audiences pénales, la consécutive en revanche semble plus indiquée pour l'entretien avec un psychologue ou un psychiatre, qui se déroule dans un tout autre contexte (dans son cabinet, ou en milieu carcéral ou hospitalier). L'interprète adapte sa traduction pour permettre à la personne de saisir le sens exact de la question (elle doit être claire, simple, sans artifice linguistique de la part du locuteur). La priorité pour le psychiatre et le psychologue est de cerner les propos de la personne au plus près de ses pensées, de son positionnement, de son vécu, de ses sentiments les plus intimes. Il convient de laisser un temps 
pour la réflexion, le silence, les pauses, les émotions; autant d'aspects qui seront ensuite exploités par le spécialiste dans son rapport. La superposition de deux discours (celui du psychiatre et celui de l'interprète) peut perturber la personne. Inévitablement, il y aura une perte de clarté. L'interprète veille à être le plus fidèle possible à la parole de la personne et, pour y parvenir, il a besoin de plus de temps pour procéder sans précipitation et en toute sérénité.

Quant au traducteur, il s'adapte à la nature du texte. Pour les actes administratifs, il fait ressortir le langage officiel de l'administration, la voix passive et le discours impersonnel (préférant «il est délivré le présent acte» à «on a délivré le présent acte»). Les actes administratifs contiennent des informations qui servent à la reconnaissance d'un droit, à l'octroi d'avantages pécuniaires, etc. Sa traduction doit être claire et précise, sans ambiguïté. Enfin, il faut être conscient de ce que le destinataire de l'acte voudra tirer le meilleur parti de toutes les informations qu'il renferme. II souhaite qu'il n'y ait pas d'implicite, ni de déduction, que tout soit dit précisément et sans interprétation de la part du traducteur afin de ne pas nuire à la compréhension du texte.

Le traducteur prend la plume du rédacteur de l'acte, il écrit comme s'il était juriste, fonctionnaire d'une administration, médecin... Chaque domaine a son langage spécifique et ses codes de rédaction.

\section{La recherche des bons indices dans la traduction des actes judiciaires}

Au tout début, lorsqu'il manque encore d'expérience dans ce type d'exercice et que nombre des termes employés lui sont inconnus, la tâche peut sembler ardue au traducteur. Pour surmonter les difficultés une à une (Irimia, 2020:35-50), développons à présent quelques démarches à effectuer:

- Tenir compte du rédacteur de l'acte: le juge (qui dit le droit) ordonne, le procureur (chargé de la prévention) requiert, l'avocat (pour la défense) soulève une nullité. Chacun utilise un langage spécifique propre à sa fonction.

- Se placer dans la phase de la procédure qui a donné lieu à la rédaction de l'acte. Une procédure se divise en effet en plusieurs étapes. La procédure pénale se 
décline ainsi en enquête policière, instruction par le juge d'instruction, phase judiciaire et d'exécution des peines. La procédure civile implique l'assignation, la mise en état, la phase judiciaire et d'exécution des jugements. À chaque étape correspond un langage spécifique. En France, le langage employé par le juge d'instruction n'est pas utilisé dans les actes d'enquête menés par le ministère public. Prenons l'exemple d'un traducteur qui doit exprimer l'idée qu'une enquête pénale a été ouverte (dans un système de droit qui ne connaît pas de juge d'instruction). Il utilisera la formule suivante: la mise en mouvement de l'action pénale par le procureur et non pas celle de mise en examen, spécifique au juge d'instruction. Plus encore, l'enquête pénale fait appel à divers langages: ceux de la preuve, des auditions, des recherches scientifiques, etc. Le jugement requiert aussi un langage spécifique (de l'instruction de l'affaire, des preuves, de la condamnation, etc.). Enfin, les termes en usage au tribunal correctionnel diffèrent de ceux de la cour d'assises, bien que les deux juridictions soient dites «de premier degré». À titre d'exemple, la personne est déclarée non coupable au tribunal de police, relaxée au tribunal correctionnel et acquittée à la cour d'assises.

- Ne pas sortir les termes de leur contexte. Prenons, par exemple, la notion de décision qui, en droit français, a un caractère générique englobant les arrêts, les jugements (sentences) et les ordonnances. Une cour d'appel rend un arrêt; un tribunal judiciaire, un jugement ou une sentence pénale; un juge d'instruction, un juge des libertés et de la détention, un juge de mise en état ou en référé, une ordonnance. Dans un autre système, cela peut être différent. À titre d'exemple, en droit roumain, la notion de décision désigne une décision d'une cour d'appel (arrêt dans la procédure française) et s'oppose à celle de sentence, rendue par une juridiction de première instance. Un mot se traduit dans le contexte de l'acte à traduire et non pas isolément, extrait d'un dictionnaire juridique. Si l'on va plus loin, un terme indique toute une procédure (par exemple, la notion de personne recherchée renvoie ainsi à la procédure de mandat d'arrêt européen ou d'extradition). 
- Chercher le concept caché derrière le terme. Par exemple, la notion de procédure simplifiée correspond en droit français à la procédure d'ordonnance pénale, mais prend une tout autre signification en droit roumain, où elle correspond à la procédure de reconnaissance préalable de culpabilité (CRPC).

\section{L'interprète face à la traduction}

Il arrive que l'interprète soit sollicité d'abord pour interpréter et ensuite pour une traduction puisque son nom figure dans la procédure. Il est couramment pensé, fût-ce inconsciemment, que l'interprète peut s'autoriser à traduire. Mais est-ce raisonnable?

Si l'on convient que traduction et interprétation sont complémentaires, on est tenté d'affirmer que l'interprète peut s'autoriser à traduire. Mais sous quelles conditions? Le champ d'action de la traduction est plus vaste que celui de l'interprétation. Vu la grande diversité des actes à traduire, le traducteur accède à des notions non accessibles à l'interprète.

À notre avis, il n'est pas raisonnable pour un interprète de traduire sur la sollicitation du tribunal quand il n'a jamais montré le moindre intérêt pour la traduction ni acquis les bases de celle-ci.

\section{Quels sont les problèmes inhérents}

\section{à la traduction judiciaire?}

Convenons-en, la plupart des traducteurs, hautement diplômés des facultés de langues, littérature et civilisation, sont à même de traduire et de faire une recherche terminologique. Ils connaissent la problématique de la traduction, que nous tâchons de synthétiser ci-après: quelle est la part de liberté du traducteur (large ou rétrécie)? La traduction consiste-t-elle à faire la copie servile du texte (le mot à mot ou le sens)? Le traducteur doit prendre en considération la langue et la culture cibles, dans un style satisfaisant à l'exactitude, au respect du public de destination et aux règles de rédaction (style soigné, grammaire, mise en page, etc.).

Toujours est-il que, lorsqu'on est amené à traduire des actes judiciaires, on entre de plain-pied dans le domaine 
de la linguistique juridique qui interfère avec le droit (qu'il faut connaitre!). Voici deux exemples de graves erreurs de traduction: la minute du jugement, qui constate par écrit la décision des juges (le dispositif) et qui n'est établie qu'en un seul exemplaire conservé par le greffe du tribunal, est prise pour le moment où le jugement a été rendu; la procédure des référés (en civil), par laquelle une partie demande au juge, en raison de l'urgence, de prendre une mesure provisoire, est assimilée à une comparution immédiate, procédure qui permet au procureur de faire juger une personne de suite après une garde à vue.

\section{Quelques aspects épineux}

de la traduction d'actes judiciaires

Fort heureusement pour les traducteurs, le langage judiciaire n'évolue que lentement par rapport à d'autres disciplines. Par ailleurs, les actes judiciaires abondent en expressions répétitives, stéréotypées, utilisées par les juges pour gagner du temps. Il est possible de s'appuyer sur des modèles d'actes, un jugement pénal de première instance rendu à Paris ressemblant à son équivalent lyonnais. Enfin, dans la procédure pénale, où le traducteur est le plus sollicité, la chaîne des actes de procédure est cohérente, et il est facile de s'y retrouver.

Les difficultés tiennent au fait que les juges ne sont pas tenus de modifier leur façon de rédiger lorsque leurs décisions seront traduites par un traducteur. Si des notions lui échappent, le traducteur doit alors se former ou consulter un spécialiste. La rédaction des décisions de justice suit un formalisme accru, le langage reste technique pour asseoir l'autorité de la justice. C'est pour cette raison que les décisions ne sont pas accessibles aux justiciables, fait qui a suscité un débat: cela concerne-t-il aussi les traducteurs? Les décisions de justice doivent-elles leur être accessibles? Avant de répondre à ces questions, il convient d'en régler une autre, celle de savoir si la relation entre un juge et un traducteur est un rapport d'initié à initié ou d'initié à non-initié. Or il nous semble qu'un juge traite plutôt le traducteur comme un initié (marque de reconnaissance). 
Néanmoins, il serait souhaitable que les avocats soignent particulièrement la rédaction des actes (conclusions ou assignations) destinés à être traduits, évitent les termes génériques, peu précis ou trop imagés, et surtout proscrivent toute imprécision, source de confusion.

Un traducteur peut ainsi être confronté à plusieurs obstacles:

- certaines énonciations n'appartiennent qu'au langage judiciaire;

- les actes judiciaires souffrent d'un certain formalisme justifié par le besoin d'apporter à l'acte une autorité suffisante et, par là même, d'asseoir l'autorité du pouvoir judiciaire (le traducteur doit faire ressortir ce formalisme dans sa traduction);

- les constructions syntaxiques, les figures de style, l'utilisation d'outils grammaticaux particuliers (notamment les connecteurs logiques ou de motivation), l'usage de modes et de temps verbaux appropriés, de constructions verbales spécifiques, les tournures impersonnelles sont des moyens mis à disposition des juges pour afficher l'autorité dont ils sont investis;

- le langage de l'obligation: les jugements relèvent du mode performatif, un ordre est donné, une obligation de faire ou de ne pas faire.

Attardons-nous, à titre d'exemple, sur l'expression de l'obligation. Elle se retrouve notamment dans le verbe du dispositif d'un jugement. C'est ce que le juge impose aux parties (en pénal, condamnation ou relaxe, en civil, une obligation de payement ou d'exécution d'une obligation).

Il existe plusieurs façons d'exprimer l'obligation:

- des tournures réclamant le subjonctif: il a été convenu que, il est impératif que;

- l'ajout d'une locution contraignante: dans tous les cas (obligation absolve), nécessairement, obligatoirement, sans restriction, sans dérogation, en aucun cas...

- des tournures impersonnelles: il convient, il appartient, il est nécessaire, il incombe (aux parties, au juge), force est de, il s'agira de...

- des verbes spécifiques: entendre, manquer, exiger, sommer de, s'engager à, devoir, se devoir de, requérir, garantir, s'interdire de, être en obligation de, être 
tenu de, être obligé de, être astreint à, avoir l'obligation de, vouloir que, exiger, revendiquer (un droit), mettre en demeure de, il faut, être redevable, être contraint, mettre à la charge de quelqu'un...

Pour les actes judiciaires, quelques aspects sont à régler au préalable par le traducteur: la connaissance des procédures qui donnent lieu aux documents à traduire, la compréhension du texte, l'extraction des mots juridiques inconnus et la recherche documentaire - qui représentent elles-mêmes une technique supposant de savoir où chercher l'information rapidement (Héba Medhat-Lecoca, 2021:177-185).

Pour la compréhension du texte, il est nécessaire de s'interroger sur les aspects suivants: qui est à l'origine de l'acte, quelles sont les fonctions ou les prérogatives de l'auteur de l'acte, à quelle étape de la procédure judiciaire l'acte a-t-il été émis? Prenons, à titre d'exemple, l'expression «où étant et parlant à...», que l'on trouve dans un acte de signification d'un huissier de justice. Ici, celui-ci notifie un acte de procédure (un jugement, une assignation, des conclusions d'avocat, etc.) : il se rend au domicile du destinataire de l'acte (où étant...) et discute avec celui-ci pour la remise de cet acte (et parlant à...). Si l'on comprend le contexte, la traduction vient d'elle-même. Toute traduction, en actes judiciaires, commence par la compréhension des problèmes de droit; ceuxci étant réglés, le traducteur pourra traduire.

\section{La nécessaire compréhension des notions de droit}

Pour ce qui est de l'interprétation, on peut croire, à tort, qu'il s'agit d'une simple mission orale visant la traduction des faits. Les interprètes qui participent aux audiences correctionnelles savent que des problèmes de droit sont soumis au débat. Comment procéder dans une audience pour une affaire de blanchiment d'argent, de banqueroute suivie de cessation de paiements et d'un plan de redressement d'une entreprise, sans avoir de notions de droit? Comment traduire à la cour d'assises la description du fonctionnement d'une arme faite par un expert en balistique sans préparation préalable? Une mission d'interprétation doit être prise au sérieux et, dans la mesure du possible, préparée. Un interprète est appelé pour une grosse affaire qui dure quelques jours. 
C'est un indice pour lui. II lui serait utile d'aller consulter l'ordonnance du juge d'instruction au greffe de la chambre correctionnelle. Pour les affaires habituelles, il demandera à l'huissier de justice ou à l'avocat la possibilité de consulter cette ordonnance le jour de l'audience avant que l'affaire ne soit appelée.

Nous admettons que l'interprète, étant davantage appelé dans les procédures pénales, apprend le droit au passage (de nombreux interprètes se sont ainsi formés sur le tas). En conséquence, ne conviendrait-il pas d'assister aux audiences correctionnelles? C'est bien là que l'on apprend véritablement, bien plus que lors d'une formation à proprement parler.

Voyons maintenant le cas du traducteur. II est appelé pour nombre de procédures civiles qui échappent à l'interprète. Elles sont nombreuses, diverses et complexes. Nous avons remarqué, en notre qualité de formatrice, que les traducteurs n'ont pas une grande affection pour le civil (excepté les jugements du droit de la famille) et qu'ils se limitent aux procédures pénales, pour lesquelles la traduction est plus facile. C'est, je crois, une erreur de leur part, dans la mesure où cela signifie qu'ils délaissent un marché important, étant donné la multitude de traductions demandée en civil par les entreprises et les particuliers.

\section{Quand I'interprétation et la traduction s'entremêlent: la traduction orale}

Un nouveau type de traduction est apparu au niveau des procédures pénales: la traduction orale d'actes écrits existant dans la procédure créée par la directive 2010/64/ UE relative au droit à l'interprétation et à la traduction du 20 octobre 2010 .

Le principe du droit à la traduction avait auparavant été posé par la Cour européenne des droits de l'homme, qui a jugé que le droit à l'interprète ne se limite pas à l'interprétation, mais s'étend aussi à la traduction de tout acte de la procédure engagée contre le prévenu, afin que celui-ci les comprenne et bénéficie d'un procès équitable.

Pour transposer en droit français la directive 2010/64/UE dans le cadre des procédures pénales, le législateur français 
a introduit, par la loi n²013-711 du 5 août 2013 portant diverses dispositions d'adaptation dans le domaine de la justice en application du droit de l'Union européenne et des engagements internationaux de la France, un paragraphe consacré au droit fondamental à l'interprétation et à la traduction (l'article préliminaire III, §2 du Code de procédure pénale et un nouvel article $n^{\circ} 803-5$ du même code).

II ressort, à la lecture de ces deux textes, une situation tout à fait originale: une mission supplémentaire de traduction à l'oral d'actes écrits est donnée à l'interprète présent dans une procédure judiciaire pour une interprétation orale. La traduction orale d'actes de procédure judiciaire écrits est un acte de traduction hybride effectué par un interprète qui, à l'origine, est désigné pour effectuer une interprétation. II sera donc invité, lorsqu'une traduction d'actes écrits est nécessaire, à la faire oralement et sur-le-champ devant la personne et devant l'autorité judiciaire qui l'a désigné, sans préparation aucune (Irimia, 2019: 57-59). Ce genre de traduction ne respecte donc pas les étapes habituelles de la traduction écrite: préparation, traduction proprement dite, correction et mise en forme. II va de soi que l'interprète ainsi désigné ne peut pas faire de recherches dans des ouvrages, des dictionnaires, ni trouver une quelconque information.

\section{Les incidences sur la qualité de la traduction}

D'abord, la traduction exécutée à l'oral ne sera certainement pas de qualité, même si elle émane de traducteurs assermentés, rompus à la traduction d'actes judiciaires, et ce, parce que les conditions d'une bonne traduction ne sont pas réunies.

Ensuite, est-il raisonnable d'attribuer une fonction bivalente d'interprète et de traducteur à un interprète qui n'est pas expert (puisque inscrit seulement sur la liste parallèle du procureur, dite CESEDA, par un tribunal judiciaire pour les procédures visant le séjour des étrangers) ou qui est seulement assermenté en tant qu'interprète auprès d'une cour d'appel alors qu'il n'est pas ou guère formé à la traduction écrite?

Les raisons de cette interrogation renvoient à l'idée que l'interprétation en audience, généralement orientée sur les faits par le biais de questions/réponses, repose en principe 
sur le biculturalisme, la plupart des interprètes étant biculturels, ou l'étant devenus. La traduction écrite d'actes de procédure est un acte intellectuel différent et difficile, car il ne suffit pas de connaître deux langues, il faut encore avoir un savoir juridique confirmé. Ce savoir est d'autant plus important que les actes à traduire sont qualifiés d'essentiels par ladite directive et que la traduction d'actes de procédure renforce le droit de la défense et garantit le respect du droit à un procès équitable.

\section{Conclusion}

Nous avons énoncé les grandes difficultés de la traduction et de l'interprétation en milieu judiciaire et nous nous sommes interrogée sur la pertinence qu'il y a à confier des traductions à un interprète qui n'est pas traducteur. La pratique à l'oral est, certes, fort utile pour la compréhension des procédures judiciaires. Néanmoins, si un interprète ambitionne de traduire, il doit (ré)examiner les diverses manières d'aborder le domaine de la traduction dans toute sa complexité. Ses seules connaissances en tant qu'interprète ne peuvent pas justifier le passage à la traduction, il en faut davantage.

\section{dorina.irimia@idtradformation.fr}

Dorina IRIMIA est traductrice agréée par la Cour de cassation et la Cour d'appel de Lyon, après une expérience en qualité d'attachée d'enseignement supérieur et d'avocate en Roumanie. Elle est titulaire d'un doctorat en droit français et, depuis 2015, exerce comme formatrice en droit et langues.

SOURCES CITÉES

BASTARD Bastard et MOUHANNA Christian, Une justice dans l'urgence, Paris, PUF, 2007.

IRIMIA Dorina, Les experts traducteurs-interprètes en milieu judiciaire, Paris, Éditions Sydney Laurent, 2021.

IRIMIA Dorina, Du langage judiciaire à la traduction. Manuel d'initiation en droit et en actes judiciaires, Paris, Éditions Sydney Laurent, 2020. 
IRIMIA Dorina, « Réflexions sur l'application effective de la directive 2010/64/UE du Parlement européen relative au droit à l'interprétation et à la traduction dans le cadre des procédures pénales", in Experts, 144, 2019, p. 57-59.

MEDHAT LECOCQ Héba, Terminologie comparée et traduction. Approche interdisciplinaire, Paris, Éditions des archives contemporaines, coll. «InterCulturel», 2021. 